\title{
Stability modeling of the LHC Nb-Ti Rutherford cables subjected to beam losses
}

\author{
L. Bottura, ${ }^{1}$ M. Breschi, ${ }^{2}$ E. Felcini, ${ }^{1, *}$ and A. Lechner ${ }^{1}$ \\ ${ }^{1}$ CERN, CH-1211 Geneva, Switzerland \\ ${ }^{2}$ University of Bologna, 40121 Bologna, Italy
}

(Received 4 February 2019; published 25 April 2019)

\begin{abstract}
The Large Hadron Collider (LHC) at CERN is being prepared for its full energy exploitation during run III, i.e., an increase of the beam energy beyond the present $6.5 \mathrm{TeV}$, targeting the maximum discovery potential attainable. This requires an increase of the operating field of the superconducting dipole and quadrupole magnets, which in turn will result in more demanding working conditions due to a reduction of the operating margin while the energy deposited by particle loss will increase. Beam-induced magnet quenches, i.e., the transition to normal conducting state, will become an increasing concern, because they could affect the availability of the LHC. It is hence very important to understand and be able to predict the quench levels of the main LHC magnets for the required values of current and generated magnetic fields. This information will be used to set accurate operating limits of beam loss, with sufficient but not excessive margin, so to achieve maximal beam delivery to the experiments. In this study we used a one-dimensional, multistrand thermal-electric model to analyze the maximum beam losses that can be sustained by the LHC magnets, still remaining superconducting. The heat deposition distribution due to the beam losses is given as an input for the stability analysis. Critical elements of the model are the ability to capture heat and current distribution among strands, and heat transfer to the superfluid helium bath. The computational model has been benchmarked against energy densities reconstructed from beam-induced main dipole quenches during LHC operation at $6.5 \mathrm{TeV}$. The model was then used to evaluate the stability margin of both main dipole and main quadrupole magnets at different beam energies, up to the expected ultimate operating energy of the LHC, 7.5 TeV. The comparison between the quench levels underlines how the increase of beam energy implies a substantial reduction of magnets stability and will require much stricter setting on the allowable beam losses to avoid resistive transitions during operation.
\end{abstract}

DOI: 10.1103/PhysRevAccelBeams.22.041002

\section{INTRODUCTION}

The magnet system of the Large Hadron Collider (LHC) [1-3] at CERN consists of about 8000 superconducting magnets of different size and field level built with approximately 1200 tons of superconducting $\mathrm{Nb}-\mathrm{Ti} / \mathrm{Cu}$ cables. The system is operated at $1.9 \mathrm{~K}$ by means of superfluid helium, which provides an efficient thermal vector for heat removal, and contributes significantly to the thermal stability of the superconducting cables.

Though carefully engineered, experience from LHC operation highlighted the importance of heat disturbances due to losses from the particle beams. Beam losses can be of different nature, ranging from halo particles and particle

\footnotetext{
*Corresponding author. enrico.felcini@cern.ch

Published by the American Physical Society under the terms of the Creative Commons Attribution 4.0 International license. Further distribution of this work must maintain attribution to the author(s) and the published article's title, journal citation, and DOI.
}

shower which are only partially intercepted by the LHC collimation system, to interaction of the primary beams with macroparticles falling into the beam chamber. The consequent release of energy in the magnet windings can lead to a quench, a resistive transition that always leads to a relatively fast temperature increase of the magnets affected, the dump of the current of the associated circuit and of course a beam dump. A comprehensive analysis of controlled quench experiments, carried out at beam energies between $450 \mathrm{GeV}$ and $4 \mathrm{TeV}$ in LHC run I, was presented in [4].

To prevent quenching, the LHC is equipped with a system of beam loss monitors (BLMs) which detects particle losses around the ring and triggers the extraction of the beams when signals exceed a prescribed threshold. The advantage of this procedure is that it avoids the lengthy cryogenic recovery that inevitably follows the resistive transition, at the price of recycling the machine, reinjecting and accelerating a new beam. Dump thresholds for the BLMs are set by comparing the particles energy deposition to the expected stability of the superconducting cables in the coil. An accurate prediction of the energy margin of the superconducting elements is hence of primary importance 
for optimizing abort thresholds and therefore improving the machine availability.

Since the publication of Ref. [4], the operational beam energy has been increased to $6.5 \mathrm{TeV}$ in LHC run II (2015-2018). In total, twelve beam-induced quenches were observed during operation at $6.5 \mathrm{TeV}$, most of them caused by beam interaction with macroparticles. In the near future, the operating energy of the LHC is expected to increase following the consolidation work planned for the second long shutdown (LS2) taking place from 2019 to 2020. An energy increase requires higher operating field and current in the main magnets, thus a reduced operating margin. At the same time, beams of higher energy are more likely to deposit more heat by beam loss mechanisms. We hence expect that the matter of magnet stability vs beam loss levels will become even more critical in the future. Indeed, this is the main motivation for the stability analyses reported here, in support and preparation of the setting of beam loss monitors for operation of the LHC from run III onwards.

In this paper we define the energy margin as the maximum energy deposition per unit volume that the magnet can withstand without undergoing an irreversible transition to the normal state (the quench mentioned earlier) under given operating conditions (current level, magnetic field). Many experimental and numerical studies have dealt with the analysis of the stability and quench of individual wires [5-10] and multistrand Rutherford cables for accelerator magnets [11-18]. Most of the analyses quoted were performed on localized heat depositions. In some investigations the impact of the heat release location on the quench propagation and development has been analyzed [19]. The peculiarity of the heat deposition from beam losses is that the disturbance is not pointlike but distributed in a nonuniform way in the magnet cross section. The work presented here considers this nonuniform heat deposition, modeled as an exponential profile to describe the radial decay of the heat load from the magnet bore to the outer part of the inner layer. This exponential model was established in [20] as a good approximation of several possible heat deposition profiles calculated with the FLUKA code [21,22].

The main magnets of the LHC are wound using Rutherford cables, made of strands compressed to form a flat two-layer cable. The polyimide-insulated cables are then wound to form the magnet coils. Superfluid helium permeates the microchannels left between the wires, and between the insulation layers, the cable interstitial space. A large helium channel, referred to here as the helium bath, is located between the magnet cold bore and the coil, providing the main contribution to the steady state cooling of the windings. Due to the proximity of superfluid helium, heat transfer mechanisms have a significant impact on the stability margin as discussed in [23]. As described more in detail later, the electrothermal response of the cable is also very relevant for stability. The model we use is based on a distributed parameter nonlinear circuit coupled with a thermal model [24,25]. All strands of the cable are considered separately in the model, taking into account the current and heat exchange between them during electrothermal transients. Finally, as we discussed in [20], we consider here the magnetic field variation along the single strands, contrasted to [12] where only the peak field was used to calculate the energy margin. In summary, the model used here contains the most advanced features that we could include.

Though the model is in essence the same as presented in [20], we will first recall its main features. We then report on the validation of the model itself, and of the parameters chosen. As a validation, we have taken the result of quench experiments performed on the main bending magnets of the LHC. The most recent energy density estimates from beam losses were used, reconstructed from beam-induced quenches during operation at $6.5 \mathrm{TeV}$. The validated model is then used to extrapolate stability margins at higher energy levels of the LHC machine, up to the expected upper energy limit for its operation of about $7.5 \mathrm{TeV}$. The stability analysis performed in this work is focused at the Rutherford cables used for the inner layer of the LHC main bending (MB) magnet and of the main quadrupole (MQ) magnet of the LHC.

\section{THE RUTHERFORD CABLE MODEL}

The cable is described through an electrothermal model governed by $1 \mathrm{D}$ equations along the longitudinal space coordinate $x$ [25]. This assumption is based on the fact that the cable length is much greater than any linear dimension in the cable cross section. The evolution of the domain state variables is described by appropriate sets of nonlinear equations for each physics domain. The equations are coupled among thermal and electric components, and solved with independent discretization in time and space. The space discretization is based on the finite element method, while the time one uses a multistep finite difference algorithm.

The electric model describes the current distribution and redistribution between strands during electrothermal transients, whereas the thermal model describes heat exchange between adjacent and nonadjacent strands, interstitial helium and the helium bath [20].

The cables considered in the analysis are the MB inner and outer layer cables, made of 28 and 36 composite $\mathrm{Nb}-\mathrm{Ti} / \mathrm{Cu}$ strands respectively, also referred to as LHC01 and $\mathrm{LHCO2}$, respectively. We recall that the MQ are wound with cables identical to the MB outer layer, i.e., LHC02, of course at different operating conditions. The main properties of the strand and Rutherford cables for the MB and MQ, taken from [26], are reported in Tables I and II.

\section{A. Electrothermal model}

The basic building block of the electromagnetic model is the composite $\mathrm{Nb}-\mathrm{Ti} / \mathrm{Cu}$ wire, described assuming that the 
TABLE I. Main dipole-inner layer-LHC01 cable data.

\begin{tabular}{lc}
\hline \hline Parameter & Value \\
\hline Width [mm] & 15.1 \\
Strand diameter [mm] & 1.065 \\
$\mathrm{Cu} /$ non-Cu ratio & 1.65 \\
Number of strands & 28 \\
Midthickness [mm] & 1.90 \\
Transposition pitch [mm] & 115 \\
\hline \hline
\end{tabular}

superconductive $\mathrm{Nb}$ - $\mathrm{Ti}$ filaments and the $\mathrm{Cu}$ stabilizer are electrically in parallel. The power law is adopted for the $E-J$ characteristics of the superconductor, with a parametrization of its critical surface described as in [27]. The current density is assumed uniformly distributed in each strand cross section. The transport current distribution and redistribution between strands occurs through distributed electrical conductances, and self and mutual inductances, according to the nonlinear electric circuit of a cable element described in [28]. The whole electrical model is obtained assembling a total of $N_{\text {strand }}$ strands, conductances and inductances becoming matrices of dimension $N_{\text {strand }}$. At the extremities of the cable, the voltage difference between all strands is set to zero, thus assuming an equipotential boundary condition. Details on the impact of the boundary conditions on current distribution in the presence of time varying external magnetic fields are reported in [28].

In the thermal model, the basic elements are the $N_{\text {strand }}$ composite strands (as for the electrical model), the interstitial helium and the large helium bath external to the coil. As for the electrical model, the strands are described as a homogeneous composite with uniform temperature on each strand cross section. Heat exchange takes place between the strands, adjacent and nonadjacent, the interstitial helium and the helium bath. The thermal components can dissipate heat due to the Joule effect, transport it by conduction and exchange it at their mutual interfaces, as described in [29]. Also in this case the interstrand thermal conductance and heat exchange coefficients yield coupling matrices among all thermal elements. Two different boundary conditions of the thermal model are set at the ends of the cable composed of $N_{\text {strand }}$ strands. On one end the strand temperature is set to a fixed value, $T_{i}=1.9 \mathrm{~K}\left(i=1, N_{\text {strand }}\right)$, representing a far end contact with the thermal reservoir of the helium

TABLE II. Main quadrupole LHC02 cable data.

\begin{tabular}{lc}
\hline \hline Parameter & Value \\
\hline Width [mm] & 15.1 \\
Strand diameter [mm] & 0.825 \\
$\mathrm{Cu} /$ non-Cu ratio & 1.95 \\
Number of strands & 36 \\
Midthickness [mm] & 1.48 \\
Transposition pitch [mm] & 100 \\
\hline \hline
\end{tabular}

bath. A sufficient length is taken (see later) not to influence the results in the heated region. On the other cable end the adiabatic condition $\frac{\partial T_{i}}{\partial x}=0\left(i=1, N_{\text {strand }}\right)$ is imposed, in order to represent the symmetry of the problem with respect to the middle point of the region heated by the thermal disturbance generated by the beam losses.

The heat transfer with the helium is described with two main mechanisms that are quite different due to the relevant timescales: the first between the strands and the interstitial helium, acting on a fast timescale, and the second between the strands and the helium bath, important for long heating durations. A transient heat transfer coefficient is taken into account in the description of the heat transfer to the interstitial helium. The model adopted in this work is described in detail in [30]. The model takes into account different phases of the interstitial helium, following its transition from He II to He I and finally to the gaseous form, as explained in $[11,12]$. The description of the heat transfer in the Kapitza phase was improved by including a crisis of the heat transfer coefficient in this phase when the heat flux reaches a given threshold of $100 \mathrm{~kW} / \mathrm{m}^{2}$. The impact on the stability margin of the parameters involved in the heat transfer model towards interstitial helium is shown in [30]. For the heat transfer coefficient towards the helium bath we use data derived from stationary heat transfer experiments [31].

\section{B. Model parameters}

The values taken for the model parameters are based as much as possible on direct observations, or matched to experimental results. At the same time, given the model completeness and complexity, it is important to realize that it is not realistic to expect a direct experimental definition of all parameters required for the complete model. This is the case, as an example, for the thermal and electrical contact resistances among strands that could appear in the model as a function of location in a cable cross section as well as longitudinal position. This level of detail cannot be measured, nor probably inferred from global cable properties. For this reason we have simplified our choice as discussed below.

The electrical contact conductances per unit length can only take two possible values, referred to as $g_{a}$ and $g_{c}$ for adjacent and nonadjacent strands respectively. In this work the conductances are taken uniform along the cable length, and are derived from the values of the electrical contact resistances between adjacent and nonadjacent strands, usually indicated in the literature as $R_{a}$ and $R_{c}$ [18]. The values of $R_{a}$ and $R_{c}$ used for the MQ inner layer cable were derived from direct measurements [32], whereas those for the MB inner layer cable were taken from [18]. These parameters are reported in Table III.

The thermal conductances between strands were determined through the knowledge of the contact surfaces between the strands, which were thoroughly analyzed in [18], 
TABLE III. Simulation parameters.

\begin{tabular}{lcc}
\hline \hline Parameter & \multicolumn{2}{c}{ Value } \\
\hline Cable type & MB (LHC01) & MQ (LHC02) \\
\hline Adjacent strands contact surface $\left[\mathrm{mm}^{2} / \mathrm{m}\right][18]$ & 371.16 & 199.68 \\
Nonadjacent strands contact surface $\left[\mathrm{mm}^{2}\right][18]$ & 1.11 & 0.66 \\
Adjacent strands thermal conductance $\left[\mathrm{W} / \mathrm{m}^{2} \mathrm{~K}\right][18]$ & 5000 & 5000 \\
Nonadjacent strands thermal conductance $\left[\mathrm{W} / \mathrm{m}^{2} \mathrm{~K}\right][18]$ & 2500 & 2500 \\
Adjacent strands electrical resistance $[\Omega][18]$ & $140 \times 10^{-6}$ & $320 \times 10^{-6}$ \\
Nonadjacent strands electrical resistance $[\Omega][18]$ & $17.5 \times 10^{-6}$ & $40.0 \times 10^{-6}$ \\
Wetted perimeter interstitial He [m] $[19,33]$ & $1.3110^{-4}$ & $0.94 \times 10^{-4}$ \\
Area of interstitial He $\left[\mathrm{m}^{2}\right][33,34]$ & $3.29 \times 10^{-6}$ & $1.73 \times 10^{-6}$ \\
\hline \hline
\end{tabular}

where they are modeled with a linear dependence on the location across the cable width, $w$.

In this work, the contact surfaces were assumed uniform and corresponding to the values obtained at the middle position of the cable width. For the adjacent strands thermal conductance, a reference value $\lambda_{a, \mathrm{MB}}=\lambda_{a, \mathrm{MQ}}=$ $5000 \mathrm{~W} / \mathrm{m}^{2} \mathrm{~K}$ was selected for both the MB and MQ cables; half of this value was taken for the nonadjacent strands [18].

The cable thermal stability strongly depends on the amount of interstitial superfluid helium, $A_{\mathrm{He}}$, and the strand perimeter wetted by direct contact, $W P_{\mathrm{He}}$. The tomographic analysis reported in [19] showed that both the interstitial helium cross section and the wet perimeter are variable across the cable width, with minimum values at the cable edges. In this work, these parameters were taken uniform along the cable width and length. In particular, only one thermal element was adopted to describe the total amount of interstitial helium. The value of the interstitial helium cross sectional area evaluated in [34] was selected here for the MB cables, resulting in $A_{\mathrm{He}, \mathrm{MB}}$ to $3.29 \times 10^{-6} \mathrm{~m}^{2}$.

As for the wetted perimeter, the average integral value of the measurements presented in [19] was computed and the proper perimeter was attributed to each strand: $W P_{\mathrm{He}, \mathrm{MB}}=$ $2.79 \times 10^{-4} \mathrm{~m}$. For the estimation of the amount of interstitial helium in the $\mathrm{MQ}$, geometric scale factors were applied, as proposed in [33]: $A_{\mathrm{He}, \mathrm{MQ}}=A_{\mathrm{He}, \mathrm{MB}} / 1.9=$ $1.73 \times 10^{-6} \mathrm{~m}^{2}$ and $W P_{\mathrm{He}, \mathrm{MQ}}=W P_{\mathrm{He}, \mathrm{MB}} / 1.4=1.99 \times$ $10^{-4} \mathrm{~m}$. These parameters are also listed in Table III. It is important to underline that the heat exchange with the interstitial helium dominates the thermal stability of the system, as explained in [30]. Therefore, the heat transfer coefficient between strands and helium, the amount of interstitial helium and its wetted perimeter with each strand, are the most crucial quantities for the model presented in this work.

\section{SIMULATION SETUP}

The simulations were performed considering only one cable at a time, i.e., neglecting the effect of neighboring cables in the coil. This is justified because the energy perturbations of interest are distributed in the coil, so that the temperature gradient between adjacent cables is small, and by the relatively long timescale of heat transfer among cables compared to the time of decision for stability. In particular, the analysis was done for the inner-layer middle plane cable, for both MB and MQ magnets. As shown by the magnetic field maps in Fig. 1, the cables in the midplane location do not experience the maximum magnetic field, located at the pole of the magnet coils for both $\mathrm{MB}$ and MQ. The cables considered, therefore, operate at higher margin than the minimum in the coil. However, as discussed in [35], the most intense beam loss heat deposition is on the magnet midplane, hence the analyzed cables are subjected to the most critical conditions.

The simulations presented here were performed on a $4 \mathrm{~m}$ long Rutherford cable, with heat deposition located in a $2 \mathrm{~m}$ long region at the middle of the cable. This is sufficiently long to avoid end effects from heat transfer at the fixed temperature boundary condition described earlier, and the heated zone is representative of the typical length scale of energy deposition due to beam loss in a magnet. Symmetry of the cable with respect to the middle point of the heated region was assumed, and represented by a zero heat flux boundary condition (see Sec. II A). As a result, only half of the cable length (i.e., $2 \mathrm{~m}$ ) requires modeling. The heat

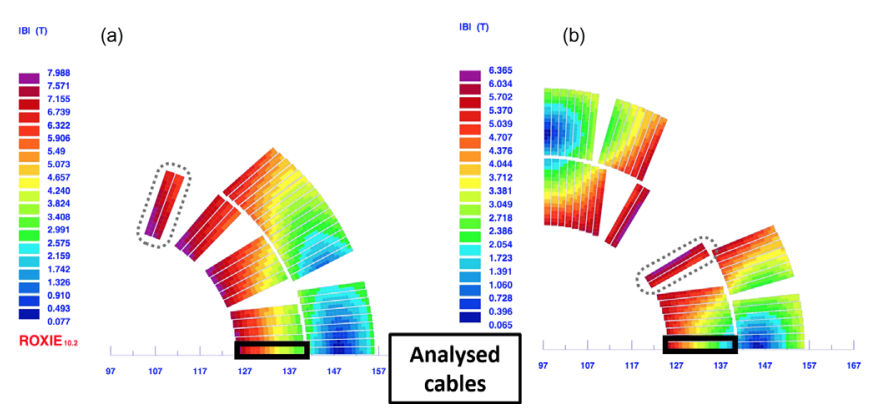

FIG. 1. Magnetic flux density in the cross section of the (a) LHC main dipole and (b) LHC main quadrupole for $6.5 \mathrm{TeV}$ of beam energy. Black rectangles indicate the analyzed cables (midplane inner layer), while the dotted lines highlight the regions of maximum field (pole). 
TABLE IV. Operating parameters of the main bending magnet middle-planer inner layer cable for different beam energies.

\begin{tabular}{lccccc}
\hline \hline $\begin{array}{l}\text { Energy } \\
{[\mathrm{TeV}]}\end{array}$ & $\begin{array}{c}T_{\text {op }} \\
{[\mathrm{K}]}\end{array}$ & $\begin{array}{c}I_{\text {op }} \\
{[\mathrm{A}]}\end{array}$ & $\begin{array}{c}B_{\max } \\
{[\mathrm{T}]}\end{array}$ & $\begin{array}{c}B_{\min } \\
{[\mathrm{T}]}\end{array}$ & $\begin{array}{c}\Delta T_{\text {min -cable }} \\
{[\mathrm{K}]}\end{array}$ \\
\hline 6.5 & 1.9 & 11000 & 7.58 & 3.00 & 2.65 \\
7.0 & 1.9 & 11850 & 8.15 & 3.21 & 2.13 \\
7.5 & 1.9 & 12750 & 8.73 & 3.42 & 1.55 \\
\hline \hline
\end{tabular}

disturbance, applied from $x=0 \mathrm{~m}$ to $x=L_{\text {cable }} / 2$, starts at $t=0 \mathrm{~s}$ and ends at $t=\tau_{Q}$.

The operating conditions of temperature, current and maximum magnetic field for the MB and MQ cables analyzed are presented in Tables IV and V, as a function of the beam energy. Note that for consistency with the assumptions of a homogeneous strand cross section, we have taken the value of magnetic field computed at the center of the strand. In its zigzag path along the cable, each strand goes from the inside of the coil, where it experiences the maximum field $B_{\max }$ of Table IV or V, to the outside location where it only sees the minimum value $B_{\min }$. The field dependence along the developed strand length has been approximated using linear piecewise interpolation.

As to the heat disturbance, its profile was computed using the FLUKA code which models energy deposition due to the interaction of particle with matter, including nuclear phenomena [21,22]. The space profile of energy deposition in the coil results in an exponential decay from its peak value at the inside, towards the minimum value in the outside. This profile in the coil thickness has been interpolated along the cable length, following again the zigzag path of a single strand in the cable. Although the heat disturbances exhibit significantly different profiles in time, as described in [4], these energy depositions are simulated here through a reference square pulse with a plateau duration set to $\tau_{Q}$.

The space dependences of both magnetic flux density and of the heat deposition are important for the accurate prediction of the stability margin. The nonuniform profile of magnetic flux density and heat deposition along the cable length resulting from the interpolations described above is shown in Fig. 2 for a selected strand. The profile has a period equal to the twist pitch $L_{p}$. The curves for all strands are obtained from the one selected in Fig. 2, shifted

TABLE V. Operating parameters of the main quadrupole magnet middle-planer inner layer cable for different beam energies.

\begin{tabular}{lccccc}
\hline \hline $\begin{array}{l}\text { Energy } \\
{[\mathrm{TeV}]}\end{array}$ & $\begin{array}{c}T_{\text {op }} \\
{[\mathrm{K}]}\end{array}$ & $\begin{array}{c}I_{\mathrm{op}} \\
{[\mathrm{A}]}\end{array}$ & $\begin{array}{c}B_{\max } \\
{[\mathrm{T}]}\end{array}$ & $\begin{array}{c}B_{\min } \\
{[\mathrm{T}]}\end{array}$ & $\begin{array}{c}\Delta T_{\text {min-cable }} \\
{[\mathrm{K}]}\end{array}$ \\
\hline 6.5 & 1.9 & 11020 & 5.45 & 1.35 & 3.08 \\
7.0 & 1.9 & 11870 & 5.87 & 1.44 & 2.70 \\
7.5 & 1.9 & 12770 & 6.35 & 1.61 & 2.84 \\
\hline \hline
\end{tabular}

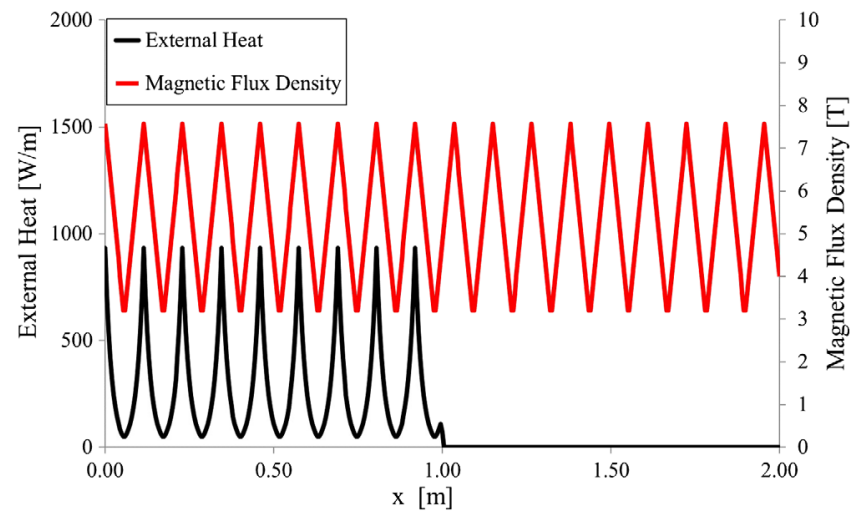

FIG. 2. Profile of the magnetic flux density and the external heat deposition along the cable length for a generic strand.

in space by the factor $L_{p}$ divided by the number of strands $N_{\text {strand }}$.

Finally, simulations of energy margin were performed by trial and error, setting a given operating condition, defining a timescale $\tau_{Q}$ for the heat deposition, and iterating on the peak heat deposition power density to find the minimum value that caused a quench (defined by a growing Joule heating at a sufficiently long time) and the maximum value that led to a recovery (defined by the disappearance of Joule heating). The energy margin was finally defined as the average of the time integral of the minimum and maximum power density waveforms. We recall that the power density has a distribution in space, and in the following we refer to the energy density that corresponds to the peak power density, i.e. the inboard side of the coil.

\section{RESULTS AND DISCUSSION}

This section reports the results of the simulations performed. First of all, we focus on the validation of the model versus reconstructed quench energies of the MB magnets operating in the LHC machine at $6.5 \mathrm{TeV}$. As a second step, the model, with unchanged parameters, is applied to the analysis of different operating currents and magnetic fields. This is the basis for the calculation of the MB and MQ cable stability at beam energies of 6.5, 7.0 and $7.5 \mathrm{TeV}$.

\section{A. Model benchmark}

The benchmark of the model is crucial for the extrapolation of the energy margin at higher energies, to gain confidence in the evaluation of the quench limits for the main LHC magnets operating beyond $6.5 \mathrm{TeV}$. For the benchmark we have used the reconstruction of the energy induced by beam interaction with macroparticles that led to magnet recovery or quench events.

Indeed, the primary cause of beam-induced magnet quenches during $6.5 \mathrm{TeV}$ operation were macroparticles interacting with the LHC proton beams [36,37]. When a 
macroparticle enters the beam tails, it gets ionized by the protons and is consequently repelled from the beam. The events typically last less than a millisecond. Despite the short loss duration, macroparticles can induce sufficient nuclear collisions to quench nearby bending dipoles. The quenches are due to secondary particle showers which heat up the coils around the midplane. In total, eight MB quenches attributed to macroparticles were observed during the $6.5 \mathrm{TeV}$ run. In addition, thousands of smaller macroparticle events were recorded by the BLMs every year, some of them causing beam aborts however without quenching magnets.

A new kind of loss events was observed in 2017 in one of the eight arc sectors of the LHC [38]. The events exhibited a similar loss signature as regular macroparticle events, but were followed by longer loss tails lasting for tens or even hundreds of milliseconds before the beams were dumped by the BLMs [39]. The events were likely caused by nitrogen or oxygen flakes in the vacuum chamber as a result of accidental air inflow in the 2016/2017 technical shutdown. It is believed that the micrometer flakes, heated up by the beam, were possibly subject to a phase transition to the gas phase, which could explain the longer loss duration. The BLM dumps prevented magnet quenches, with the exception of one case which resulted in the quench of a dipole after around 45 milliseconds.

Macroparticles represent an almost pointlike source of beam losses, which facilitates the reconstruction of such events. Using BLM measurements, the collision vertex and the number of proton-nucleus collisions can be determined by means of shower simulations [39]. The results presented in the following were calculated with the FLUKA code.

Figure 3 compares the reconstructed energy densities with predictions of the electrothermal model. The plot includes loss events with and without a subsequent magnet quench, covering the time spectrum from submilliseconds

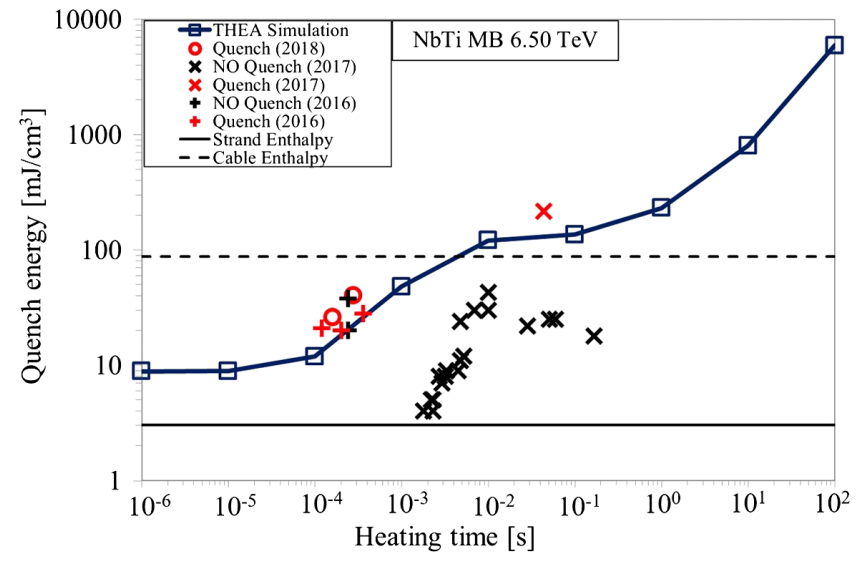

FIG. 3. Quench energy of the LHC main bending magnet at 6.5 TeV, as a function of the heat pulse duration, in comparison with reconstructed energy densities for beam loss events observed in the 2016-2018 proton runs. to a few tens of milliseconds. The events without quench provide a lower limit of the minimum quench energy density, whereas those with quench indicate an upper limit. The systematic error of the reconstructed energy densities is estimated to be about a factor of 2. This includes the uncertainty in the number of proton-nucleus collisions, but also approximations in the geometry model and uncertainties in the interaction models. In one of the cases, around $0.25 \mathrm{~ms}$ timescale, no quench occurred at an estimated energy density which is about a factor 2 higher than that of an event at similar timescale, leading to a quench. The reason for this outlier is not fully understood, as in general the typical uncertainty is expected to be the same for different events.

As can be seen in Fig. 3, the calculations performed with the proposed electrothermal model are consistent with a recovery or quench in all events apart from the outlier. The data gathered in the disturbance time range above $1 \mathrm{~ms}$ exhibit a significant gap between the recovery and quench cases, which prevents a more precise validation of the model for such loss durations. Still, the estimated energy densities for recovery and quench events with submillisecond duration are very close to each other.

At longer timescale, the model shows an increase of stability at around $10 \mathrm{~ms}$ that is consistent with the recoveries observed at an energy density higher than the quench limits for fast energy deposition. As we discussed elsewhere [11] the increase of the energy margin in this timescale is related to transfer of heat and current among the strands, a very delicate matter in the model, which seems nonetheless to capture the actual magnet response.

An additional important remark is that in general the temporal profile of macroparticle energy deposition resembles a skew normal distribution, or can even have a more irregular shape in case the macroparticle is subject to a phase transition. Despite the general approach of assuming a rectangular time pulse, the developed electrothermal model is able to estimate and predict without free parameters the minimum quench energy of the LHC MB magnets subjected to beam losses.

\section{B. Main dipole (MB)—Beyond 6.5 TeV}

The model validated using the reconstructed energy densities at $6.5 \mathrm{TeV}$ was used to explore the dependence of the energy margin of the MB magnets as a function of beam energy. This was done modifying the operating conditions of current and field, while maintaining all other model features and parameters. The operating currents and magnetic flux density fields corresponding to the different beam energies are reported in Table IV.

As expected, higher beam energies result in more demanding operating conditions for the magnets (hence for the cables) and results in a reduction of the thermal stability of the system, as presented in Fig. 4. 


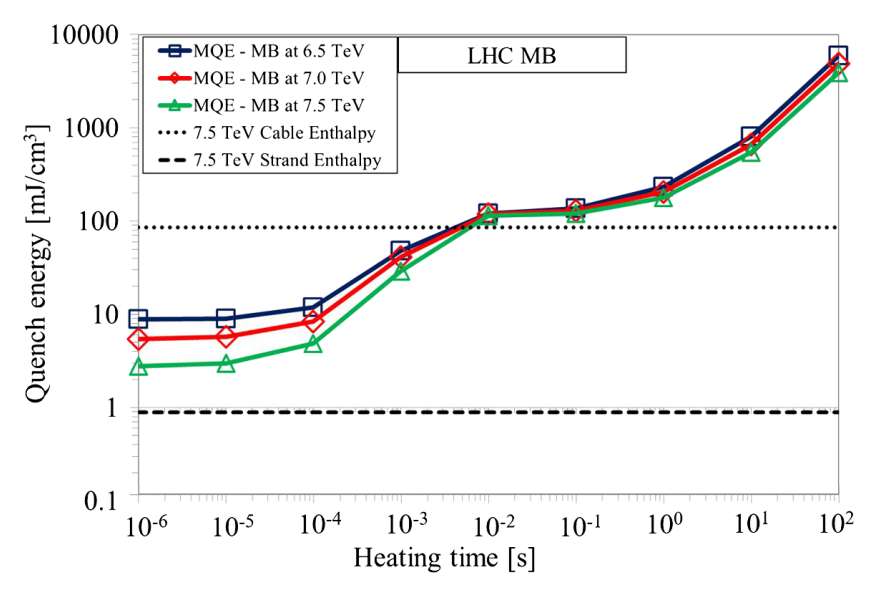

FIG. 4. Quench energy of the LHC main bending magnet, as a function of the heat pulse duration, at the different operating conditions required by the beam energy. The dashed lines represent the enthalpy of the cable and the strand.

The plot shows the energy margin evaluated at $6.5,7$ and 7.5 $\mathrm{TeV}$ as a function of the timescale of the energy deposition, and also reports the enthalpy of both the composite strands $(\mathrm{Nb}-\mathrm{Ti}$ and $\mathrm{Cu})$ and the cable $(\mathrm{Nb}-\mathrm{Ti}$, $\mathrm{Cu}$ and interstitial $\mathrm{He}$ ), computed between the operating temperature $T_{\mathrm{op}}$ and the minimum value of $T_{\text {cs-cable }}$ on the analyzed cable at $7.5 \mathrm{TeV}$. The operating temperature $T_{\mathrm{op}}$, set to $1.9 \mathrm{~K}$, is the undisturbed operating temperature and $T_{\text {cs-cable }}$ is the current sharing temperature of the innermost strand of the cable, which is subjected to the highest value of magnetic field and heat deposition, and thus exhibits the lowest $T_{\text {cs-cable }}$. The cable minimum temperature margins, $\Delta T_{\text {min-cable }}=T_{\text {cs-cable }}-T_{\text {op }}$, are listed in Table IV.

We note how the increase of operating current and field result in different responses in terms of stability for fast and slow transients [40]. In the timescale of microseconds, the energy is absorbed locally, by the heat capacity of the cable and interstitial helium. Because heat capacity is very small at low temperature, and grows rapidly as the temperature increases, the effect of a reduction of temperature margin is very strong. On the other hand, for very slow to steady state energy inputs, the timescale of second, the dominating mechanism is heat transfer to the helium bath. This is also a highly nonlinear mechanism, very effective under a small temperature difference between the strand and helium, a few tens of $\mathrm{mK}$, but rapidly saturating as the temperature difference increases. In this case a reduction of the temperature margin only has a marginal effect, as observed. In the intermediate timescale, around the millisecond, heat and current redistribution between strands is important and we note a transition from single strand to a collective response.

The dependencies of quench energy discussed above are presented in Fig. 5 as a function of the minimum temperature margin in the coil, i.e. $\Delta T_{\min \text {-coil }}=T_{\text {cs-coil }}-T_{\text {op }}$, where $T_{\text {cs-coil }}$ is the current sharing temperature at the location of the coil peak field, namely the pole, as shown in Fig. 1.

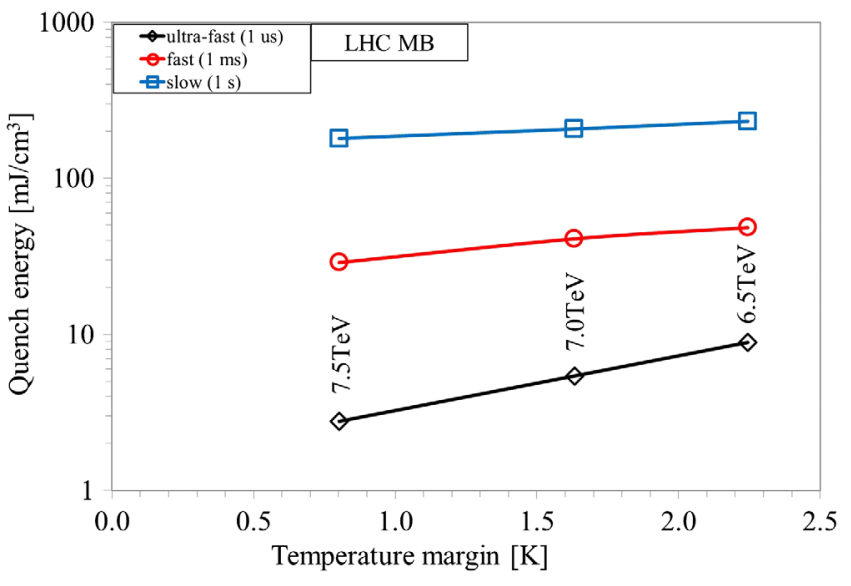

FIG. 5. Quench energy of the LHC main bending magnet, as a function of the minimum temperature margin on the coil relative to the beam energy, at the different heat pulse time durations.

In this form we see more clearly the effect of different mechanisms of heat exchange at fast and slow heat deposition timescales. In the first case, ultrafast timescale, the energy margin is reduced by a factor 3 when operation at $7.5 \mathrm{TeV}$ is compared to $6.5 \mathrm{TeV}$. This matches well to the decrease of the temperature margin, as we see that the value of $\Delta T_{\text {min-coil }}$ at $7.5 \mathrm{TeV}$ is slightly above $0.8 \mathrm{~K}$, to be compared to about $2.3 \mathrm{~K}$ at $6.5 \mathrm{TeV}$. For longer heat deposition, on the slow timescale, the energy margin drops by about $20 \%$ from 6.5 to $7.5 \mathrm{TeV}$.

It is clear from this analysis that operation of LHC at higher beam energy will call for a delicate balance of beam intensity vs loss, and effective beam loss detection to prevent magnet quench.

\section{Main quadrupole (MQ)-Beyond 6.5 TeV}

As for the MB analysis, we have applied the model to estimate the quench energy values of the main quadrupole magnets of the LHC machine. We recall here that the MQ cable is LHC02, with an increased number of smaller diameter strands. The model parameters have been adapted to take this into account. As shown graphically in Fig. 1 and reported in Table $\mathrm{V}$, the peak field on the inner-layer middle plane cable of the MQ is significantly lower than in MB, with similar operating current, resulting in higher temperature margin $\Delta T_{\text {min-cable. }}$. As expected, the MQ exhibits higher values of quench energies in the whole range of operating conditions analyzed. The results are presented in Fig. 6, together with the strand and cable minimum enthalpy at $7.5 \mathrm{TeV}$.

Coherently with the previous studies, beam energy increase implies relevant reduction of the minimum energy required to quench the magnet. The different phases of heat exchange and current distribution can be identified at different timescales, similarly to the MB. The minimum expected energy margin, for fast energy deposition and operation at $7.5 \mathrm{TeV}$ is nonetheless quite large, just below 


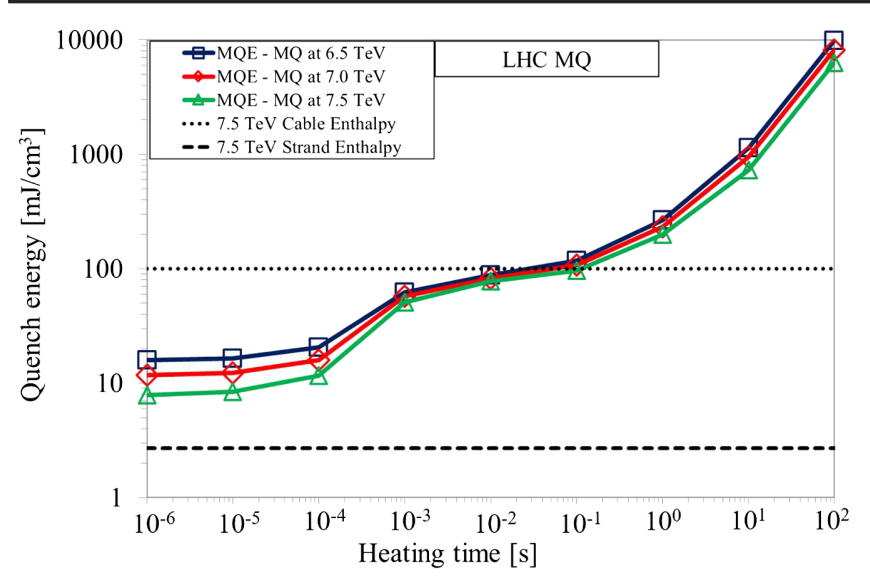

FIG. 6. Quench energy of the LHC main quadrupole magnet, as a function of the heat pulse duration, at the different operating conditions required by the beam energy. The dashed lines represent the enthalpy of the cable and the strand.

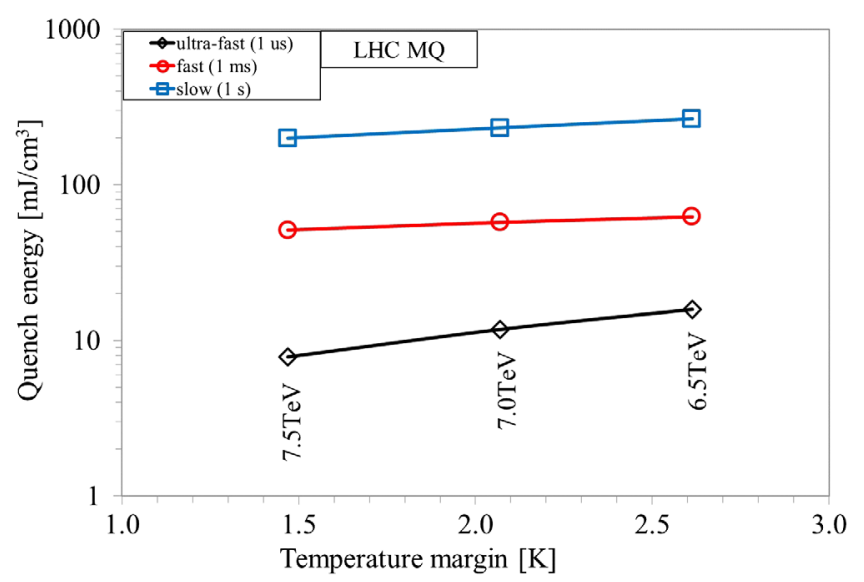

FIG. 7. Quench energy of the LHC main quadrupole magnet, as a function of the minimum temperature margin on the coil relative to the beam energy, at the different heat pulse time durations.

$10 \mathrm{~mJ} / \mathrm{cm}^{3}$, to be compared to values of less than $2 \mathrm{~mJ} / \mathrm{cm}^{3}$ obtained in the MB. Given this result, we do not expect that the MQ will be limiting.

Finally, Fig. 7 presents the quench energies as a function of the minimum temperature margin in the coil, analogous to Fig. 5 for the MB, for operating conditions corresponding to $6.5,7.0$ and $7.5 \mathrm{TeV}$ beam energy. In this case, for $7.5 \mathrm{TeV}$, the $\Delta T_{\text {min-coil }}$ is about $1.5 \mathrm{~K}$, which is significantly greater than the minimum margin for the MB mentioned earlier.

\section{CONCLUSIONS}

In this paper we have reviewed the features of a multistrand thermoelectric model applicable to the Rutherford cables used in the LHC. The model, presented in detail elsewhere, has been adapted to the analysis of the LHC main magnets electrothermal stability in case of energy deposition following beam losses.

Reconstructed energy densities for beam losses in the main bending magnet operating at beam energy of $6.5 \mathrm{TeV}$ were used to benchmark the model and its crucial features and parameters, mainly the heat transfer from strands to helium, the amount of interstitial helium in direct contact with the strands and the corresponding wetted perimeter. We have shown by direct comparison that the computed energy margin is consistent with the measured quench limits in a broad range of timescales, from fraction of millisecond to several tens of milliseconds.

The selection of the model parameters was based on direct observation or matched to experimental results. The validation versus stability margin values reconstructed from the machine operation make the model a suitable tool to estimate the minimum quench energies of the LHC magnets subjected to beam losses, in view of a future beam energy increase up to the ultimate value of $7.5 \mathrm{TeV}$.

The validated model was hence applied to the calculation of the minimum quench energy of both the main bending dipoles and the main quadrupoles, at $6.5,7.0$ and $7.5 \mathrm{TeV}$, in order to explore the effect of the increase of operating current and field in view of a full energy exploitation of the LHC.

As expected, we found that an increase of the beam energy, accompanied by the corresponding increase of the operating current and field, leads to considerable reduction of the energy margin for fast timescales: a factor 3 from 6.5 to $7.5 \mathrm{TeV}$. Interestingly, the decrease of energy margin is not as dramatic for slow timescales, limited to a $20 \%$ drop from 6.5 to $7.5 \mathrm{TeV}$. The difference of behavior could be attributed to the change in mechanisms that dominate heat transfer at different timescales. We have also shown that the main bending dipoles are by far the magnets that will be most affected by the increased beam energy.

From the analysis results, it is clear that machine operation beyond $6.5 \mathrm{TeV}$ will pose unprecedented challenges. One possible threat for the future machine performance could be beam interactions with macroparticles, which were already the main cause of beam-induced quenches in run II as shown in this paper. Because of the lower quench margin at 7 and $7.5 \mathrm{TeV}$, even smaller sized macroparticles can lead to a quench. Beam loss monitor measurements recorded in run II, together with the considerations in this paper, indicate that the number of macroparticle events, which can possibly induce a dipole quench, increases by about a factor of 2-4 if the beam energy increases from 6.5 to $7 \mathrm{TeV}$. It is however difficult to make absolute predictions about the expected number of quenches as this depends on the long-term evolution of such events. Besides the events attributed to macroparticles, also other kinds of beam losses were observed in run II. These included for example losses caused by a macroscopic obstacle in one of the main bending magnets or, in other cases, by a sudden $10 \mathrm{~Hz}$ oscillation of the beam. It can also not be excluded that 
new types of beam losses occur in the future. The methods and results presented here will be useful to adapt the machine protection systems for such loss events, and in particular forecast the setting of beam loss monitors whose function is to prevent magnet quenches.

\section{ACKNOWLEDGMENTS}

We acknowledge the support of Susana Izquierdo Bermudez from CERN for providing $\mathrm{MB}$ and $\mathrm{MQ}$ field maps.

[1] O. Bruning et al., LHC design report, CERN Report No. CERN-2004-003, 2004.

[2] L. Rossi, The LHC superconducting magnets, in Proceedings of the 21st Particle Accelerator Conference, Knoxville, TN, 2005 (IEEE, Piscataway, NJ, 2005), pp. 141-145.

[3] G. Annala, P. Bauer, L. Bottura, M. A. Martens, N. Sammut, G. Velev, and V. Shiltsev, Advances in the understanding and operations of superconducting colliders, in Proceedings of the 21st Particle Accelerator Conference, Knoxville, TN, 2005 (Ref. [2]), pp. 54-58.

[4] B. Auchmann et al., Testing beam-induced quench levels of LHC superconducting magnets, Phys. Rev. ST Accel. Beams 18, 061002 (2015).

[5] K. Seo, M. Morita, S. Nakamura, T. Yamada, and Y. Jizo, Minimum quench energy measurements for superconducting wires, IEEE Trans. Magn. 32, 3089 (1996).

[6] D. E. Baynham, D. A. Cragg, D. C. Coombs, P. Bauer, and R. Wolf, Transient stability of LHC strands, IEEE Trans. Appl. Supercond. 9, 1109 (1999).

[7] P. Bauer, R. Wolf, L. Oberli, and M. N. Wilson, Minimum quench energies of LHC strands, IEEE Trans. Appl. Supercond. 9, 1137 (1999).

[8] F. Trillaud, F. Ayela, M. Fratini, D. Leboeuf, and P. Tixador, A novel technique for minimum quench energy measurements in superconductors using a single mode diode laser, Cryogenics 45, 585 (2005).

[9] M. Breschi, L. Trevisani, L. Bottura, A. Devred, and F. Trillaud, Comparing the thermal stability of NbTi and $\mathrm{Nb}_{3}$ Sn wires, Supercond. Sci. Technol. 22, 025019 (2009).

[10] M. Breschi, L. Trevisani, M. Boselli, L. Bottura, A. Devred, P. L. Ribani, and F. Trillaud, Minimum quench energy and early quench development in $\mathrm{Nb}$-Ti superconducting strands, IEEE Trans. Appl. Supercond. 17, 2702 (2007).

[11] L. Bottura, M. Calvi, and A. Siemko, Stability analysis of the LHC cables, Cryogenics 46, 481 (2006).

[12] P. P. Granieri, M. Calvi, P. Xydi, B. Baudouy, D. Bocian, L. Bottura, M. Breschi, and A. Siemko, Stability analysis of the LHC cables for transient heat depositions, IEEE Trans. Appl. Supercond. 18, 1257 (2008).

[13] M. Breschi, P. P. Granieri, M. Calvi, M. Coccoli, and L. Bottura, Quench propagation and stability analysis of Rutherford resistive core cables, Cryogenics 46, 606 (2006).
[14] G. P. Willering, A. P. Verweij, J. Kaugerts, and H. H. J. ten Kate, Stability of Nb-Ti Rutherford cables exhibiting different contact resistances, IEEE Trans. Appl. Supercond. 18, 1263 (2008).

[15] A. Devred, Investigation of the normal zone along a superstabilized superconducting solenoid, J. Appl. Phys. 66, 2689 (1989).

[16] M. N. Wilson and R. Wolf, Calculation of minimum quench energies in Rutherford cables, IEEE Trans. Appl. Supercond. 7, 950 (1997).

[17] D. Bocian, B. Dehning, and A. Siemko, Modeling of quench limit for steady state heat de- posits in LHC magnets, IEEE Trans. Appl. Supercond. 18, 112 (2008).

[18] G. Willering, Stability of superconducting Rutherford cables for accelerator magnets, Ph.D. thesis, University of Twente, 2009.

[19] G. P. Willering, A. Verweij, C. Scheuerlein, A. den Ouden, and H. H. ten Kate, Difference in stability between edge and center in a Rutherford cable, IEEE Trans. Appl. Supercond. 18, 1253 (2008).

[20] M. Breschi, A. Bevilacqua, L. Bottura, and P. P. Granieri, Analysis of beam-induced quenches of the LHC cables with a multistrand model, IEEE Trans. Appl. Supercond. 25, 4700405 (2015).

[21] T. T. Böhlen, F. Cerutti, M. P. W. Chin, A. Fassò, A. Ferrari, P. G. Ortega, A. Mairani, P. R. Sala, G. Smirnov, and V. Vlachoudis, The FLUKA code: Developments and challenges for high energy and medical applications, Nucl. Data Sheets 120, 211 (2014).

[22] A. Ferrari, P. R. Sala, A. Fassò, and J. Ranft, FLUKA: A multiparticle transport code, Reports No. CERN-2005-10, 2005, No. INFN/TC_05/11, and No. SLAC-R-773.

[23] P. P. Granieri, Heat transfer between the superconducting cables of the LHC accelerator magnets and the superfluid helium bath, Ph.D. thesis, EPFL, Lausanne.

[24] A. Akhmentov, L. Bottura, M. Breschi, and P. L. Ribani, A theoretical investigation on current imbalance in flat two superconducting cables, Cryogenics 40, 627 (2000).

[25] L. Bottura, C. Rosso, and M. Breschi, A general model for thermal, hydraulic and electric analysis of superconducting cables, Cryogenics 40, 617 (2000).

[26] J. D. Adam, Status of LHC superconducting cables mass production, CERN LHC Project Report No. 549, 2002.

[27] L. Bottura, A practical fit for the critical surface of Nb-Ti, IEEE Trans. Appl. Supercond. 10, 1054 (2000).

[28] L. Bottura, M. Breschi, and A. Musso, Calculation of interstrand coupling losses in superconducting Rutherford cables with a continuum model, Cryogenics 96, 44 (2018).

[29] M. Breschi, E. Felcini, and L. Bottura, Quench level of the HL-LHC $\mathrm{Nb}_{3} \mathrm{Sn}$ IR quadrupoles, IEEE Trans. Appl. Supercond. 27, 4002105 (2017).

[30] M. Breschi, E. Felcini, F. Breccia, P. P. Granieri, E. Bergonzoni, A. Bevilacqua, P. Galassi, T. Winkler, and L. Bottura, Modeling of beam loss induced quenches in the LHC main dipole magnets, IEEE Trans. Appl. Supercond., 29, 4004407 (2019).

[31] P. P. Granieri and R. Weelderen, Deduction of steady-state cable quench limits for various electrical insulation 
schemes with application to LHC and HL-LHC magnets, IEEE Trans. Appl. Supercond. 24, 4802806 (2014).

[32] D. Richter (private communication).

[33] J. Depond, D. Leroy, and L. Oberli, Superconducting cable topology, main magnets and superconductors group LHCMMS, Internal note No. 97-09.

[34] T. Winkler, Helium II heat transfer in LHC magnets, Ph.D. thesis, University of Twente, 2017.

[35] M. Massimini, Electrical and thermal analysis of superconducting wires and LHC cables, Master's thesis, University of Bologna, 2012.

[36] G. Papotti et al., Macroparticle-induced losses during 6.5 TeV LHC operation, in Proceedings of the 7th International Particle Accelerator Conference (IPAC18), Busan, South Korea, 2016, pp. 1481-1484, https://cds.cern $. \mathrm{ch} / \mathrm{record} / 2293680 ? \mathrm{ln}=\mathrm{en}$.

[37] A. Lechner et al., BLM thresholds and UFOs, in Proceedings of the 7th Evian Workshop on LHC Beam Operation, Evian
Les Bains, France, 2016, pp. 209-214, http://accelconf.web .cern.ch/AccelConf/ipac2018/papers/mopmf053.pdf.

[38] J. M. Jiménez et al., Observations, analysis and mitigation of recurrent LHC beam dumps caused by fast losses in arc half-cell 16L2, in Proceedings of the 9th International Particle Accelerator Conference IPAC18, Vancouver, Canada, 2018, pp. 228-231.

[39] A. Lechner, B. Auchmann, E. Bravin, A. A. Gorzawski, L. K. Grob, E. B. Holzer, B. Lindstrom, T. Medvedeva, D. Mirarchi, R. Schmidt, M. Valette, and D. Wollmann, beam loss measurements for recurring fast loss events during 2017 LHC operation possibly caused by macroparticles, in Proceedings of the 9th International Particle Accelerator Conference IPAC18, Vancouver, Canada, 2018, pp. 780-783, http://inspirehep.net/record/1690712/files/ tupaf040.pdf.

[40] L. Bottura, Modeling stability in superconducting cables, Physica (Amsterdam) 310C, 316 (1998). 